\title{
Use of Syndromic Surveillance Information for Expanded Assessment of Wildfire Disaster
}

\author{
Jeffrey Johnson*1, Michele Ginsberg ${ }^{1}$, Nancy French ${ }^{2}$, Brian Thelen ${ }^{2}$ and Benjamin Koziol ${ }^{2}$ \\ ${ }^{1}$ San Diego County Public Health Services, San Diego, CA, USA; ${ }^{2}$ Michigan Tech Research Institute, Ann Arbor, MI, USA
}

\section{Objective}

This presentation describes how syndromic surveillance information was combined with fire emission information and spatio-temporal fire occurrence data to evaluate, model and forecast climate change impacts on future fire scenarios.

\section{Introduction}

Syndromic surveillance information can be a useful for the early recognition of outbreaks, acute public health events and in response to natural disasters. Inhalation of particulate matter from wildland fire smoke has been linked to various acute respiratory and cardiovascular health effects. Historically, wildfire disasters occur across Southern California on a recurring basis. During 2003 and 2007, wildfires ravaged San Diego County and resulted in historic levels of population evacuation, significant impact on air quality and loss of lives and infrastructure. In 2011, the National Institutes of HealthNational Institute of Environmental Health Sciences awarded Michigan Tech Research Institute a grant to address the impact of fire emissions on human health, within the context of a changing climate. San Diego County Public Health Services assisted on this project through assessment of population health impacts and provisioning of syndromic surveillance data for advanced modeling.

\section{Methods}

Various historical data sets were used during this study. These included: emergency department syndromic surveillance from 17 hospitals, as well as air quality and particulate matter, meteorological, wildland burn fuel, and atmospheric dispersion data. The study area was San Diego County. These data were linked temporally and spatially to create statistical models based on selected modeling approaches including generalized additive modeling. Future fire frequency was modeled for the entire region to determine the impacts of climate change on future fire and health outcomes. Modeling based upon previous fire occurrence was used to develop models for future fire indexes, risk of ignition, potential burning, and fire weather.

\section{Results}

Several models were developed to produce expected respiratory health impacts under future climate conditions for the San Diego County region. Model results showed that at peak fire par-ticulate concentrations, the odds of a person seeking emergency care in- creased by approxi-mately $50 \%$ compared to non-fire conditions. Also developed was a model to forecast future fire occurrence based on regional climate model predictions. This forecast covering the next the next three decades reveals that San Diego County will experience approximately two extreme fire seasons each decade by 2040 .

\section{Conclusions}

Syndromic surveillance data are useful during disasters for situational awareness. These data may also provide value for post-disaster analytic work and predictive modeling for future disas-ters. This study demonstrates utility of syndromic data for collaborative work resulting in better understanding of environmental interactions on human health. We do know that wildfire oc-curs with some degree of historic regularity and these results may be useful for preparedness planning. We also know the conditions which must be present for medium to high wildland fire impact upon the population. This study further supports the notion that agencies should be able to strategically deploy resources and messaging immediately preceding the fire period with the goal of reducing human health risk factors and encouraging changes in community behaviors before, during and after a fire. Future fire model shows San Diego County will experience approximately two extreme fire seasons each decade by 2040 . This project also promoted collaboration between public health and environmental health entities to better understand de-terminants of health during a disaster. In addition to developing a better understanding of the consequences of climate change on fire-induced respiratory illness, the project funding has provided support for San Diego County to improve their syndromic surveillance capacity and infrastructure.

\section{Keywords}

Syndromic; Disaster; Forecasting; Wildfire; San Diego

\section{Acknowledgments}

This work has been supported by the NIH-NIEHS Climate Change Grant \#1RC1ES018612-01. We would also like to acknowledge the research teams at Michigan Tech Research Institute, Michigan Technology University, and the University of Maryland Department of Geography.

\footnotetext{
*Jeffrey Johnson

E-mail: jeffrey.johnson@sdcounty.ca.gov
} 